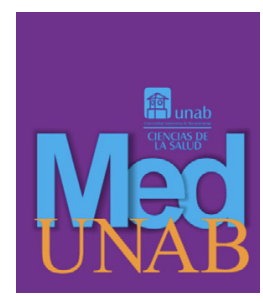

REVISTA DE LA FACULTAD

DE CIENCIAS DE LA SALUD

\title{
Hallazgos colonoscópicos en pacientes atendidos en una institución de salud del municipio de Barrancabermeja, Santander
}

Colonoscopy findings in patients attended at a health institution in the municipality of Barrancabermeja, Santander

Achados de colonoscopia em pacientes atendidos em uma instituição de saúde do município de Barrancabermeja, Santander

Nefi H Rodriguez L, Est. ${ }^{1} \mathbb{D}$, Dikson J Logreira G, Est. $^{1} \mathbb{D}^{\mathbb{D}}$, Diego F Sanabria L, Est. $^{1} \mathbb{D}^{\mathbb{D}}$, Juan C Uribe Caputi, MD, Esp. ${ }^{2}$ (D.

1. Estudiante de Medicina. Facultad de Ciencias de la Salud. Universidad De Santander UDES. Bucaramanga, Santander. Colombia.

2. Médico, Magíster en Epidemiología, Facultad de Ciencias de la Salud, Universidad De Santander UDES. Bucaramanga, Santander. Colombia.

Correspondencia. Nefi Rodriguez. Universidad De Santander UDES. Bucaramanga, Santander. Colombia. Dirección: Calle 105 número 42a -09. Barrio San Bernardo. Floridablanca, Santander. Colombia. Teléfono: 3045967722. Email: Nefi0831@gmail.com

\section{INFORMACIÓN DEL ARTÍCULO:}

Artículo recibido: 24 de enero 2020

Artículo aceptado: 28 de abril 2020

DOI: https://doi.org/10.29375/01237047.3834

Cómo citar: Rodriguez NH, Logreira JD, Sanabria DF, Uribe JC. Hallazgos colonoscópicos en pacientes atendidos en una institución de salud del municipio de Barrancabermeja, Santander. MedUNAB. 2020;23(2): 261-269. doi: 10.29375/01237047.3834

RESUMEN
Introducción. La colonoscopia es una exploración visual de la mucosa del colon,
la cual consiste en la introducción de una sonda óptica flexible por el ano. Dentro
de sus objetivos está la localización de lesiones de una manera muy precisa,
obtener biopsias para su análisis al microscopio, realizar diversos procedimientos
terapéuticos o diagnosticar lesiones pequeñas no detectables por métodos
imagenológicos tradicionales. El objetivo del presente artículo es describir los
hallazgos colonoscópicos en pacientes adultos de una institución prestadora de


servicios de salud. Metodología. Estudio observacional, transversal con componente analítico, la información se recolectó de manera retrospectiva, en pacientes mayores de 18 años en ambos sexos, a los cuales se les realizó colonoscopia en una institución prestadora de servicios de salud de segundo nivel. Resultados. Se practicaron 521 procedimientos colonoscópicos en 291 mujeres y 230 hombres con mediana de edad de 63 años (rango 18-99), la proporción fue bastante superior en las personas mayores de 50 años, la frecuencia del procedimiento en mujeres fue superior con un $57.0 \%$ vs. el $43 \%$ de los hombres. El número de colonoscopias con resultado normal, fue más frecuente en las personas menores de 50 años (54.8\%) contra $45.2 \%$ del total de los de 50 años en adelante. Conclusiones. La colonoscopia es un instrumento de gran utilidad al momento de diagnóstico, seguimiento y tratamiento de una gran variedad de patologías gastrointestinales, ya que, a través de esta importante herramienta diagnóstica es posible determinar y descartar otro tipo de patologías que afectan el sistema gastrointestinal.

Palabras claves:

Colonoscopía; Tamizaje Masivo; Gastroenterología; Neoplasias Intestinales; Intestino Delgado; Intestino Grueso.

\section{ABSTRACT}

Introduction. Colonoscopy is a visual exploration of the colonic mucosa, which consists of the insertion of a flexible optic catheter through the anus. Its objectives include to locate lesions very precisely, to obtain biopsies for their microscopic analysis, to carry out different therapeutic procedures and to diagnose small lesions that cannot be detected by traditional imaging techniques. The aim of this article is to describe the colonoscopy findings in adult patients of a health care institution. Methodology. Observational, cross-sectional study with an analytical component. The information was collected retrospectively from patients aged over 18 years, of both sexes, on which a colonoscopy was performed in a secondary health care institution. Results. A total of 521 colonoscopy procedures were conducted on 291 women and 230 men with an average age of 63 years (age range: 18-99 years). The proportion was greater among people aged over 50 years. The frequency of the procedure in women was higher with $57.0 \%$, compared to $43 \%$ in men. The number of colonoscopies with a normal result was more frequent in people aged under 50 years $(54.8 \%)$ compared to $45.2 \%$ of the total of those aged 50 years and over. Conclusion. Colonoscopy is a very useful instrument when diagnosing, monitoring and treating a great variety of gastrointestinal pathologies, because through this important diagnostic tool, it is possible to determine the existence of or rule out other kinds of pathologies that affect the gastrointestinal system.

Keywords:

Colonoscopy; Mass Screening; Gastroenterology; Intestinal Neoplasms; Small Intestine; Large Intestine.

\section{RESUMO}

Introdução. A colonoscopia é uma exploração visual da mucosa do cólon, que consiste na introdução de uma sonda óptica flexível através do ânus. Entre seus objetivos está a localização de lesões de maneira muito precisa, a obtenção de biópsias para análise ao microscópio, a realização de vários procedimentos terapêuticos ou o diagnóstico de pequenas lesões não detectáveis pelos métodos tradicionais de imagem. O objetivo deste artigo é descrever os achados de colonoscopia em pacientes adultos de uma instituição prestadora de serviços de saúde. Metodologia. Em estudo observacional, transversal, com componente analítico, as informações foram coletadas retrospectivamente em pacientes acima de 18 anos de ambos os sexos, submetidos à colonoscopia em instituição prestadora de serviços de saúde de segundo nível. Resultados. Foram realizados 521 procedimentos de colonoscopia em 291 mulheres e 230 homens com mediana de idade de 63 anos (variação de 18 a 99), a proporção foi muito maior em pessoas acima de 50 anos, a frequência do procedimento em mulheres foi maior em 57\% vs. 43\% nos homens. O número de colonoscopias com resultado normal foi mais frequente em pessoas com menos de 50 anos (54.8\%) versus $45.2 \%$ do total das pessoas com 50 anos ou mais. Conclusão. A colonoscopia é um instrumento muito útil no diagnóstico, monitoramento e tratamento de uma ampla variedade de patologias gastrointestinais, 
pois, por meio desta importante ferramenta de diagnóstico, é possível determinar e descartar outros

tipos de patologias que afetam o sistema gastrointestinal.

Palavras-chave:

Colonoscopia; Programas de Rastreamento; Gastroenterologia; Neoplasias Intestinais; Intestino

Delgado; Intestino Grosso.

\section{Introducción}

La colonoscopía es una exploración visual de la mucosa del colon (intestino grueso), que consiste en la introducción de una sonda óptica flexible por el ano que posteriormente se hace progresar a lo largo del colon, incluyendo el último tramo del intestino delgado. Dentro de sus objetivos está la localización de lesiones de una manera muy precisa, permitiendo obtener biopsias para su análisis al microscopio (1). También es posible realizar procedimientos terapéuticos tales como: extirpación de pólipos (pequeños tumores benignos y a veces también malignos), coagulación de lesiones sangrantes o extracción de cuerpos extraños, diagnosticar lesiones pequeñas no detectables por radiografía, registrar con sondas adecuadas la motilidad del colon, hacer estudios directos de las secreciones para estudios coproparasitológicos, así como determinar la verdadera extensión de los procesos inflamatorios crónicos, agudos, difusos o segmentarios (2).

Por lo anterior, es fundamental previa a su realización, una preparación de buena calidad que permita la exploración detallada de la superficie del colon, y que lleve a la detección temprana y el tratamiento preventivo de lesiones $(3,4)$. Los eventos adversos (EA) posteriores a la ingestión de preparaciones intestinales son poco frecuentes, pero pueden ser graves (5).

Los criterios propuestos por el Panel Europeo sobre la Adecuación de la Endoscopia Gastrointestinal (EPAGE I) se crearon en 1998 y se han utilizado en estudios científicos para evaluar la idoneidad de las indicaciones endoscópicas. Estos criterios se actualizaron en 2008 (criterios EPAGE II) con base en la mejor evidencia científica (6).

La colonoscopía está indicada para evaluar los signos y síntomas de sospecha de cáncer colorrectal, sangrado rectal, cambios en el hábito intestinal, dolor abdominal, anemia, etc., que son motivos comunes para la consulta de atención primaria y generalmente son causados por afecciones benignas limitadas (7). El manejo adecuado de los pacientes que presentan estos síntomas debe incluir una evaluación inicial adecuada y la consideración de los parámetros costo beneficio para el paciente.
Por otro lado, el comportamiento de las enfermedades gastrointestinales varía constantemente debido a múltiples factores, algunos relacionados con el paciente, y otros con el ambiente, la familia y la cultura. Dichos cambios implican el aumento de algunas enfermedades y la disminución de otras (8).

Los problemas del sistema digestivo afectan a personas de todas las edades, algunos de estos ocurren con mayor frecuencia en las mujeres que en los hombres. La dieta es considerada un factor importante como desencadenante de síntomas gastrointestinales cuya fisiopatología incluye desde reacciones inflamatorias (con manifestaciones claramente medibles) hasta trastornos funcionales (en los que no se ha podido demostrar o medir efecto orgánico alguno) (9).

La incidencia estimada de cáncer de colon y recto en el mundo para ambos géneros en el año 2012 fue de $17.2 \times 100,000$ personas $(1,360,602$ casos $)$, lo cual representa el $9.7 \%$ de todos los casos nuevos de cáncer diagnosticados en el mundo. Esta cifra ubica este tipo de cáncer en el cuarto lugar de incidencia después del cáncer de mama, próstata y pulmón (10). La tasa estandarizada de mortalidad para el cáncer de colon y recto en el mundo para hombres y mujeres, se estimó en el 2012 en 8.4 x 100,000 personas (693,933 casos), ubicándose así en el quinto lugar entre todas las muertes ocasionadas por cáncer (11).

El sexo masculino y la raza negra, también se asocian con una mayor incidencia y mortalidad por cáncer colorrectal. Los adultos negros tienen las tasas más altas de incidencia y mortalidad en comparación con otros subgrupos raciales o étnicos (12).

En Colombia, según los datos de la cuenta de alto costo en el año 2015, el cáncer de colon y recto ocupó el puesto número 5 entre los tumores invasivos más prevalentes en la población, con un total de 10,208 pacientes afectados por esta neoplasia; se encontró que el $89.7 \%$ de los casos correspondían a pacientes mayores de 45 años, siendo esta la población más afectada. Se presentaron un total de 1,967 pacientes con diagnóstico "de novo" de cáncer de colon y recto, lo cual representa el $6.4 \%$ de todas las incidencias. La prevalencia país fue de 21.2 por 100,000 habitantes, la 
incidencia país fue de 4.1 por 100,000 habitantes. El total de pacientes fallecidos con diagnóstico de cáncer de colon y recto fue de 1,436 , con una mortalidad país de 3 por 100,000 habitantes. En este sentido, se puede hablar de una alta carga de enfermedad en el sistema de salud, a nivel económico y social, generada por estos tipos de cáncer (13). La senescencia aumenta los riesgos de enfermedades inflamatorias del intestino y cáncer de colon (14).

En el Área Metropolitana de Bucaramanga (AMB), durante el periodo 2003-2007 y de acuerdo con datos obtenidos del Registro poblacional de cáncer del Área metropolitana de Bucaramanga (RPC-AMB), el cáncer colorrectal ocupó el tercer puesto en orden de frecuencia para ambos sexos; en hombres fue precedido por cáncer de próstata y estómago, y en mujeres por cáncer de mama y cuello uterino, con tasas de incidencia de 13.3 y de 12.2 casos/100,000 respectivamente (15). Por esta razón, las solicitudes de colonoscopía han aumentado significativamente en los últimos años como consecuencia de la eficacia mostrada en el cribado de cáncer colorrectal (CCR) $(16,17)$.

Otros factores que contribuyen al aumento del uso de la colonoscopía incluyen los siguientes: 1) marcada mejora en la comodidad y la calidad del procedimiento, principalmente debido al uso de la sedación y al advenimiento de las nuevas tecnologías; 2) implementación progresiva de programas de detección de cáncer colorrectal; y 3) el desarrollo de unidades de endoscopia de acceso abierto (18). En la actualidad se considera la prueba de elección en pacientes de alto riesgo (antecedentes familiares de CCR y/o antecedentes personales de adenomas avanzados) y es la prueba de confirmación diagnóstica en los casos de sangre oculta positiva (19).

La información presentada en este artículo, permite analizar los parámetros que se evalúan dentro del procedimiento colonoscópico y la gran importancia que se tiene a la hora de realizar el diagnóstico y/o manejo de cada una de las patologías identificadas, ya que, al ser un estudio observacional, transversal con componente analítico, permite una aproximación y es de gran utilidad para el personal asistencial, para planificar estrategias de salud encaminadas a mejorar la calidad de vida en pacientes con este tipo de alteraciones. Así mismo, es punto de partida para el diseño de nuevas preguntas de investigación, en torno a una visión multidimensional de las enfermedades colónicas.

Este artículo tiene como finalidad describir los hallazgos colonoscópicos en pacientes adultos sometidos a la realización de este procedimiento en una institución prestadora de servicios de salud en el municipio de Barrancabermeja, Santander, desde enero del año 2018 hasta abril del 2019.

\section{Metodología}

Estudio observacional, transversal con componente analítico. La información se recolectó de manera retrospectiva, la población estuvo constituida por todos los pacientes mayores de 18 años de ambos sexos, a quienes se les realizó colonoscopía de forma ambulatoria o con hospitalización, en una institución prestadora de servicios de salud de segundo nivel, entre los meses de enero de 2018 y abril de 2019. De esta población se seleccionaron 521 pacientes, 230 del sexo femenino y 291 del sexo masculino. Para la obtención de los datos necesarios se revisaron los informes colonoscópicos, tomando las variables disponibles y que se tuvieron en cuenta como edad, sexo y el diagnóstico respectivo. Las variables cualitativas (sexo y diagnóstico) se presentaron en forma de frecuencias y porcentajes y las cuantitativas en medianas y rangos, aunque la edad se categorizó en menores de 50 años y de 50 años en adelante. Para el análisis bivariado se utilizó la prueba de ji cuadrado (test $\mathrm{x} 2$ ) y en caso necesario el test exacto de Fisher; los cálculos se realizaron con el software Stata v14 (Stata $C)$.

Como criterios de inclusión se tuvieron en cuenta ser mayor de edad, vivir en la ciudad de Barrancabermeja, corregimientos y municipios aledaños del Magdalena Medio; se excluyeron los pacientes con información incompleta para los fines del estudio.

De acuerdo con la Resolución 8430 de 1993, del Ministerio de Salud de Colombia, este estudio cumple con los criterios de investigación en salud y por lo tanto está sujeto a las normas éticas correspondientes; sin embargo, se considera una investigación sin riesgo, por lo cual no requirió aprobación del Comité de Ética de la institución prestadora de servicios de salud, ya que dicho protocolo no constituye un riesgo para los participantes. Por lo cual, la institución prestadora de servicios de salud en donde se obtuvo la información de los pacientes manifestó a los investigadores que, de acuerdo a los estatutos de la institución, el estudio no debía pasar por Comité de Ética de investigación ni solicitar consentimiento informado. Adicional a esto, no se tomaron datos de identificación, ni se revelaron los datos específicos de los mismos. 


\section{Resultados}

Dentro de las principales características generales de la población, la mediana de la edad de los pacientes fue de 63 años (rango 18-99). Al categorizar la variable edad en pacientes menores de 50 años y de 50 años en adelante, la proporción de personas evaluadas fue bastante superior en los mayores $(78.7 \%$ IC95\% 75.282.2); por su parte, la frecuencia del procedimiento en mujeres fue superior con un 55.9\% (IC95\% 51.6-60.1) (Tabla 1).

Tabla 1. Características población sometida a colonoscopías. Estudio descriptivo. IPS Barrancabermeja 20182019. Variables cuantitativas y variables demográficas

Variables cuantitativas

\begin{tabular}{|c|c|c|c|c|c|}
\hline CARACTERÍSTICAS & $\mathbf{N}$ & PROM & DS & ME & Rango \\
\hline \multicolumn{6}{|l|}{ Generales } \\
\hline Edad (años) & 521 & 60.5 & 14.9 & 63 & $18-99$ \\
\hline \multicolumn{6}{|l|}{ Variables demográficas } \\
\hline CARACTERÍSTICAS & $\mathbf{N}$ & $\%$ & & IC $95 \%$ & \\
\hline \multicolumn{6}{|l|}{ Género } \\
\hline Femenino & 291 & 55.9 & & $51.6-60.1$ & \\
\hline Masculino & 230 & 44.1 & & $39.9-48.4$ & \\
\hline \multicolumn{6}{|l|}{ Edad } \\
\hline Menores de 50 años & 111 & 21.3 & & $17.8-24.8$ & \\
\hline 50 años y más & 410 & 78.7 & & $75.2-82.2$ & \\
\hline
\end{tabular}

Fuente: Tabla elaborada por los autores

La mayor parte de los pacientes remitidos para la realización del procedimiento, proceden del servicio de consulta externa con un $97.5 \%$; los dos principales diagnósticos fueron hemorroides $(65.3 \%$ IC95\% 61.269.4) y enfermedad diverticular (12.9\% IC95\% 10.0 15.7), tanto en hombres como en mujeres; mientras que el tercer diagnóstico sí fue diferente, siendo colitis en los hombres y polipectomía en las mujeres. Se encontraron diferencias significativas por sexo en los diagnósticos de enfermedad diverticular, polipectomía y proctitis, siendo estos diagnósticos proporcionalmente más frecuentes en hombres $(\mathrm{p}<0.05)$; por su parte, el resultado normal de la colonoscopía también resultó diferente por sexo, siendo más frecuente en mujeres $(\mathrm{p}<0.05)$ (Tabla 2).
Por su parte, por grupos de edad, los principales diagnósticos en menores de 50 años fueron las hemorroides (62.2\% del total en este grupo de edad) y la colitis $(5.4 \%$ del total en esta edad), aunque el segundo resultado mayormente reportado en ellos fue normal $(15.3 \%$ del total del grupo); por otro lado, en los de 50 años en adelante los más frecuentes fueron hemorroides, enfermedad diverticular y los pólipos $(66.1 \%, 15.4 \%$ y $4.9 \%$ del total en este grupo de edad). Se encontraron diferencias significativas por grupo de edad en los diagnósticos de enfermedad diverticular y colitis, siendo estos diagnósticos proporcionalmente más frecuentes en los de 50 años en adelante $(\mathrm{p}<0.05)$; por su parte, el resultado normal de la colonoscopía también resultó diferente por grupo de edad, siendo más frecuente en jóvenes, como era de esperarse $(\mathrm{p}<0.05)$ (Tabla 3$)$. 
Tabla 2. Diagnósticos reportados población sometida a colonoscopía y asociación exploratoria (test x2) entre sexo y diagnóstico. Estudio descriptivo. IPS Barrancabermeja 2018-2019

\begin{tabular}{|ccccccc}
\hline VARIABLE & $\mathbf{N}$ & $\mathbf{\%}$ & $\mathbf{I C} \mathbf{9 5 \%}$ & Mujeres (\%) & Hombres (\%) & VALOR P \\
\hline Hemorroides & 340 & 65.3 & $61.2-69.4$ & $199(58.5)$ & $141(41.5)$ & 0.092 \\
\hline $\begin{array}{c}\text { Enfermedad } \\
\text { diverticular }\end{array}$ & 67 & 12.9 & $10.0-15.7$ & $30(44.8)$ & $37(55.2)$ & 0.050 \\
\hline Normal & 31 & 5.9 & $3.9-8.0$ & $26(83.9)$ & $5(16.1)$ & 0.001 \\
\hline Pólipos & 23 & 4.4 & $2.6-6.2$ & $7(30.4)$ & $16(69.6)$ & 0.012 \\
\hline Sin diagnóstico & 18 & 3.5 & $1.9-5.0$ & $11(61.1)$ & $7(38.9)$ & 0.648 \\
\hline Colitis & 14 & 2.7 & $1.3-4.1$ & $10(71.4)$ & $4(28.6)$ & 0.284 \\
\hline Masa a estudio & 9 & 1.7 & $0.6-2.8$ & $3(33.6)$ & $6(66.7)$ & 0.192 \\
\hline Proctitis & 9 & 1.7 & $0.6-2.8$ & $1(11.1)$ & $8(88.9)$ & 0.012 \\
\hline Fisura anal & 4 & 0.8 & $0.2-1.5$ & $2(50.0)$ & $2(50.0)$ & 0.813 \\
\hline Otros diagnósticos & 6 & 1.2 & $0.2-2.1$ & $2(33.3)$ & $4(66.7)$ & 0.413 \\
\hline
\end{tabular}

Fuente: Tabla elaborada por los autores

Tabla 3. Asociación exploratoria (test $\mathrm{x} 2$ ) entre edad y diagnóstico. Estudio descriptivo. IPS Barrancabermeja 2018-2019

\begin{tabular}{ccccccc}
\hline VARIABLE & $\mathbf{N}$ & $\mathbf{\%}$ & IC 95\% & $\begin{array}{c}\text { Menor a 50 } \\
\text { años (\%) }\end{array}$ & $\begin{array}{c}\mathbf{5 0} \text { años en } \\
\text { adelante (\%) }\end{array}$ & VALOR P \\
\hline Hemorroides & 340 & 65.3 & $61.2-69.4$ & $69(20.3)$ & $271(79.7)$ & 0.440 \\
\hline $\begin{array}{c}\text { Enfermedad } \\
\text { diverticular }\end{array}$ & 67 & 12.9 & $10.0-15.7$ & $4(6.0)$ & $63(94.0)$ & 0.001 \\
\hline Normal & 31 & 5.9 & $3.9-8.0$ & $17(54.8)$ & $14(45.2)$ & 0.000 \\
\hline Pólipos & 23 & 4.4 & $2.6-6.2$ & $3(13.0)$ & $20(87.0)$ & 0.439 \\
\hline Sin diagnóstico & 18 & 3.5 & $1.9-5.0$ & $4(22.2)$ & $14(77.8)$ & 0.923 \\
\hline Colitis & 14 & 2.7 & $1.3-4.1$ & $6(42.9)$ & $8(57.1)$ & 0.046 \\
\hline Masa a estudio & 9 & 1.7 & $0.6-2.8$ & $1(11.1)$ & $8(88.9)$ & 0.692 \\
\hline Proctitis & 9 & 1.7 & $0.6-2.8$ & $3(33.3)$ & $6(66.7)$ & 0.410 \\
\hline Fisura anal & 4 & 0.8 & $0.2-1.5$ & $2(50.0)$ & $2(50.0)$ & 0.201 \\
\hline Otros diagnósticos & 6 & 1.2 & $0.2-2.1$ & $2(33.3)$ & $4(66.7)$ & 0.612
\end{tabular}

Fuente: Elaborada por los autores

\section{Discusión}

En los últimos años se ha visto un incremento en las solicitudes de realización de colonoscopía con un fin diagnóstico, terapéutico o para el seguimiento de algunas patologías. Dentro de los resultados del estudio se encontró que las personas mayores de 50 años fueron las que se sometieron mayormente a la realización de la colonoscopía (78.7\%). Aunque para el análisis de este estudio no se tuvo en cuenta la razón por la cual se indicó este examen, sí se evidencia que una de las principales razones de su solicitud fue la tamización de 
cáncer colorrectal, el cual, según recomendaciones de las distintas guías europeas y del American College of Gastroenterology, se debe realizar en personas mayores de 50 años (20).

Sin embargo, el equipo de trabajo de servicios preventivos de Estados Unidos concluyó con alta certeza que la detección del cáncer colorrectal en adultos asintomáticos de riesgo promedio de 50 a 75 años es de beneficio neto sustancial (es decir, el beneficio menos los daños). La detección del cáncer colorrectal es una estrategia de salud preventiva sustancialmente infrautilizada en Estados Unidos (21).

En un reporte de 10 años sobre colonoscopías hechas entre los años 2000 y 2010 por miembros del Servicio de Gastroenterología Hospital Clínico Universidad de Chile, de un total de 2,700 colonoscopías realizadas en pacientes hospitalizados o ambulatorios, 1,566 correspondieron a mujeres (58\%) y el rango de edad fue de 15 a 98 años con una media de 56 años. Por su parte, los motivos más frecuentes por los cuales se solicitó el procedimiento fueron el dolor abdominal $(25 \%)$ y el sangrado (20\%); la mayor parte de las colonoscopías fueron normales $(40 \%)$, entre las enfermedades mayormente reportadas están la enfermedad diverticular y los pólipos (22).

Es importante resaltar el número de colonoscopías realizadas durante estos 10 años, ya que en nuestra población del municipio de Barrancabermeja el número de colonoscopías realizadas es alto para corresponder a un solo año, lo cual podría deberse al aumento de la tendencia de solicitudes de colonoscopías.

Por otro lado, la mayor parte de estos exámenes fueron enviados por el servicio de consulta externa, donde se demostró que muchos pacientes reportan alguna sintomatología en común, teniendo como diagnóstico principal hemorroides y similares con un $65.9 \%$ (IC95\% 62.1-69.6), que se manifiestan de forma más prevalente en mujeres con un $58.7 \%$ y personas mayores de 50 años con un 79.7\%. Según European Medical Journal (EMJ), esta enfermedad afecta al $40-50 \%$ de la población en algún momento de su vida y en la mayoría de los países. Cerca del $4 \%$ de la población precisa de cirugía. Antes de empezar el tratamiento para las hemorroides con síntomas análogos como el sangrado, debe efectuarse la exclusión de una posible neoplasia (23).

Dentro del presente estudio también encontramos diferencias significativamente altas con respecto a la enfermedad diverticular (12.7\%), siendo más frecuente en hombres $(54.4 \%)$ y personas mayores de 50 años
(79.7\%). Estos divertículos son protrusiones saculares (herniaciones) de la mucosa y submucosa a través de la capa muscular del colon; estas saculaciones miden habitualmente entre 5 y $10 \mathrm{~mm}$, aunque en ocasiones pueden alcanzar los $20 \mathrm{~mm}$ (24). Según el consenso de la World Gastroenterology Organization Practice Guidelines, la enfermedad diverticular es más frecuente en hombres en poblaciones menores de 50 años, pero cuando la población se encuentra entre los 50 y 70 años, la literatura muestra que se nivelan, con un predominio leve en mujeres (25). La evaluación de más de 300,000 colonoscopías realizada por 136 gastroenterólogos mostró que cada aumento de $1.0 \%$ en la llamada tasa de detección de adenoma se asoció con una disminución de $3.0 \%$ en el riesgo de cáncer (razón de riesgo 0.97; IC 95\% 0.96-0.98). Las tasas de ausencia o no detección de adenoma durante la colonoscopía son un problema grave, ya que se estima que se pasan por alto entre el 6 y $27 \%$, lo que varía dependiendo de la técnica y el lugar donde se realizan (26).

\section{Conclusión}

La colonoscopía es un procedimiento diagnóstico invasivo de gran utilidad, factible y seguro al momento del diagnóstico, seguimiento y tratamiento de una gran variedad de patologías intestinales, principalmente en paciente con alto riesgo de aparición de cáncer colorrectal. Los resultados del estudio nos permiten abarcar otro tipo de patologías en la edad adulta; demuestran que las patologías más frecuentes son las hemorroides y similares, manifestándose de forma más prevalente en las mujeres. La fisura anal es la que en menor porcentaje se encuentra en una proporción igual en ambos sexos. También se pudo identificar que el mayor número de lesiones se encuentran en la población mayor de 50 años.

Aunque del total de pacientes analizados, el 90.6\% presentan alguna anormalidad evidenciada en el resultado de la colonoscopía, no fue posible relacionarlas con la razón médica de su solicitud, por lo que se hace necesario la realización de estudios similares en los que se pueda verificar esta información que complemente los hallazgos aquí descritos. De esta manera, se contribuye a continuar reconociendo que esta herramienta diagnóstica es necesaria para el diagnóstico temprano de patologías intestinales y colorrectales, disminuyendo la incidencia de morbimortalidad, principalmente la causada por el cáncer colorrectal, gracias al diagnóstico temprano, el tratamiento oportuno y el seguimiento colonoscópico de las lesiones precursoras. 
La limitación más relevante de este estudio fue el hecho de no tener acceso a la historia clínica completa de los pacientes, y el no conocer el motivo de consulta por el cual se les indicó la realización de la colonoscopía, lo que genera un potencial sesgo para identificar otras variables que permitieran realizar un análisis más detallado de la información.

Se recomienda continuar realizando estudios que permitan identificar las patologías más frecuentes que afectan al colon en las diferentes regiones de nuestro país, con el fin de generar intervenciones y estrategias masivas de tamizaje que permitan establecer un control oportuno de estas afecciones, ya que actualmente estas patologías presentan una incidencia ascendente con mayor impacto en la población mayor de 50 años.

\section{Confidencialidad de los datos}

Los autores declaran que en este artículo no aparecen datos de pacientes.

\section{Conflicto de intereses}

Ninguno.

\section{Agradecimientos}

A la enfermera profesional Tatiana Cala Ramírez, por su apoyo en la realización de este estudio.

\section{Referencias}

1. Colombia, Ministerio de Salud y Protección Social. Guía de Práctica Clínica para la detección temprana, diagnóstico, tratamiento integral, seguimiento y rehabilitación del cáncer de colon y recto. 2 da edición. Guía No. 20 [Internet]. Bogotá D.C.: Ministerio de Salud y Protección Social. 2017. Recuperado a partir de: gpc.minsalud.gov.co

2. García E, Martínez J, García J, Hernández M, et al. Enfermedades del colon más frecuentes en nuestro medio. Terapéutica endoscópica. Revista Mexicana de Coloproctología [Internet]. 2011. [citado 15 de octubre. de 2019] Recuperado a partir de: https://www. medigraphic.com/pdfs/proctologia/c-2011/c111b.pdf

3. Polanco Lozano JF, Reyes JJ, Pino RH, Torres L, Vallejo L. Preparación colónica en la Unidad de Endoscopia Del Hospital Universitario Hernando Moncaleano Perdomo, experiencia de un año: serie de casos. Revista De La Facultad De Ciencias De La Salud De La Universidad Del Cauca. 2016;18(2), 1016. Recuperado a partir de https://revistas.unicauca. edu.co/index.php/rfcs/article/view/140

4. Baxter NN, Goldwasser MA, Paszat LF, Saskin R, Urbach DR, et al. Association of Colonoscopy and Death From Colorectal Cancer. Ann Intern Med. 2009; 24(2): 1-8. https://doi.org/10.7326/0003-4819150-1-200901060-00306

5. Nagata N, Niikura R, Aoki T, Moriyasu S, Sakurai T, Shimbo T, et al. Risk factors for adverse in-hospital outcomes in acute colonic diverticular hemorrhage. World Journal of Gastroenterology. 2015; 21(37): 1069710703. https://doi.org/10.3748/wjg.v21.i37.10697

6. Burnand B, Harris JK, Wietlisbach V, Froehlich F, Vader JP, Gonvers JJ. Impact of colonic cleansing on quality and diagnostic yield of colonoscopy: the European Panel of Appropriateness of Gastrointestinal Endoscopy European multicenter study. Gastrointest Endosc. 2006; 63: 1018-1026. https://doi.org/10.1016/ S0016-5107(04)02776-2

7. Appropriate use of gastrointestinal endoscopy. American Society for Gastrointestinal Endoscopy. Gastrointest Endosc. 2000;52(6):831-837. https://doi. org/10.1016/S0016-5107(00)70219-7

8. Chey WD. The role of food in the functional gastrointestinal disorders: Introduction to a manuscript series. Am J Gastro-enterol. 2013 [citado el 20 de octubre de 2019]. Recuperado a partir de: https:// www.ncbi.nlm.nih.gov/pubmed/23545712

9. Moctezuma C, Aguirre J. Enfermedades gastrointestinales y hepáticas. Gaceta Médica de México. 2016 [citado el 22 de octubre de 2019]. Recuperado a partir de: http://www.anmm.org.mx/GMM/2016/s1/ GMM 152 2016 S1 074-083.pdf

10. Ferlay J, Shin HR, Bray F, Forman D, Mathers C, Parkin DM. Estimates of worldwide burden of cancer in 2008: GLOBOCAN 2008. Int J Cancer. 2010;127(12):28932917. Doi: https://doi.org/10.1002/ijc. 25516

11. GLOBOCAN-International Agency for Research on Cancer. Estimated Cancer. Incidence, mortality and prevalence worldwide in 2012. 2015. Recuperado a partir de: http://globocan.iarc.fr/Pages/ fact sheets population.aspx

12. Howlader N, Noone AM, Krapcho M, et al. SEER Cancer Statistics Review, 1975-2013. Instituto Nacional del Cáncer. 2016 [citado el 20 de septiembre de 2019].Recuperado a partir de: http://seer.cancer. gov/csr/1975 2013/.

13. Acuña L, Sánchez P, Valencia O, Arevalo I. Consenso basado en evidencia, indicadores de gestión del riesgo en pacientes con cáncer gástrico y cáncer de colon y recto en Colombia. Fondo Colombiano de Enfermedades de Alto Costo. 2017. Recuperado a partir de: https:// 
cuentadealtocosto.org/site/publicaciones/consensobasado-en-evidencia-indicadores-de-gestion-delriesgo-en-pacientes-con-cancer-gastrico-y-cancer-decolon-y-recto-en-colombia/

14. Watanabe K, Ikuno Y, Kakeya Y. et al. Age-related dysfunction of the DNA damage response in intestinal stem cells. Inflamm Regener. 2019;39,8. https://doi. org/10.1186/s41232-019-0096-y

15. Uribe-PérezCJ,Blanco-Quintero JJ, Bello-Zapata LM. Incidencia de cáncer de colon y recto en Bucaramanga, Colombia 2008-2012. MedUNAB. 2019;22(1):16-23. Doi: https://doi.org/10.29375/01237047.2711

16. Shaukat A, Mongin SJ, Geisser MS, et al. Long-term mortality after screening for colorectal cancer. N Engl J Med. 2013;369(12):1106-1114. doi:10.1056/ NEJMoa1300720

17. Nishihara $\mathrm{R}, \mathrm{Wu} \mathrm{K}$, Lochhead $\mathrm{P}$, et al. Long-term colorectal-cancer incidence and mortality after lower endoscopy. N Engl J Med. 2013;369(12):1095-1105. doi:10.1056/NEJMoa1301969

18. Fernández-Esparrach G, Gimeno-García AZ, Llach $\mathrm{J}$, et al. Recomendaciones de utilización de la endoscopia: análisis de la probabilidad de encontrar lesiones significativas en los pacientes procedentes de la atención extrahospitalaria. Med Clin (Barc). 2007; 129:205-8. Doi: https://doi.org/10.1157/13107917

19. Castellls A, Marzo M,Amador FJ, García MA, Beceiro B, Fernández A, Ferrándiz J, Giráldez D, Gonzalez V, Martínez RJ, Mascort J, Quintero E. Guía de práctica clínica de prevención de cáncer colorrectal. Guía de práctica clínica. Actualización 2009. Barcelona. Asociación Española de Gastroenterología, Sociedad Española de Medicina de Familia y Centro Cochrane Iberoamericano. 2009. Programa de guías de práctica clínica en enfermedades digestivas, desde la atención primaria a la especializada: 4. Recuperado a partir de: https://www.aegastro.es/sites/default/files/archivos/ guia-clinica/gpc colorrectal 2009.pdf

20. Geçim R, Fazal A, Khan A, Arshad M, Cheema M, Hayat W, Khan W, Farag A. Manejo de la enfermedad hemorroidal. Resumen descriptivo de presentaciones escogidas entre las ofrecidas en el Seminario Enfermedad venosa crónica y enfermedad hemorroidal: manejo y tratamiento, celebrada en Lisboa, Portugal, los días 23 y 24 de septiembre de 2016. [Internet]. 2016 [citado el 6 de octubre de 2019]. Recuperado a partir de: https://emj.europeanmedicalgroup.com/wp-content/uploads/sites/2/2018/02/ Manejo-de-la-Enfermedad-Hemorroidal.pdf.

21. Bibbins-Domingo K, Grossman DC, et al. Screening for Colorectal Cancer: US Preventive Services Task Force Recommendation Statement. JAMA. 2016 Aug 2;316(5):545. Doi: https://doi.org/10.1001/ jama.2016.5989
22. Gunther A, Abedrapo M, Rodrigo M, Ricardo C, José B, Berger. Colonoscopia: puesta al día y reporte de 10 años de experiencia. Revista Chilena de Cirugía. 2012; 64: 306-311. Doi: https://doi.org/10.4067/ S0718-40262012000300016.

23. Goodwin JS, Singh A, Reddy N, Riall TS, Kuo YF. Uso excesivo de la colonoscopia de detección en la población de Medicare. Arch Intern Med. 2011; 171(15): 1335-1343.

24. Young-Fadok TM, Roberts PL, Spencer MP, et al. Enfermedad diverticular del colon. Curr Probl Surg. 2000; [Internet]. 2000 [citado el 6 de octubre de 2019]. Recuperado a partir de: https://www.aegastro. es/sites/default/files/archivos/ayudas-practicas/38 Enfermedad diverticular.pdf

25. Ortiz H. Guía de Cirugía Colorrectal de la AEC. Madrid. Ed. Arán. 2012. Recuperado a partir de: https://www.aecirujanos.es/files/documentacion/ documentos/cirugia-colorrectal-2-edic.pdf

26. Allescher HD, Weingart V. Optimizing Screening Colonoscopy: Strategies and Alternatives. Visc Med. 2019;35(4):215-225.Doi: https://doi.org/10.1159/000501835 